\title{
PRPH2 Activates Hippo Signalling and Suppresses the Invasion and Anoikis Inhibition of Laryngeal Cancer
}

This article was published in the following Dove Press journal:

Cancer Management and Research

\author{
KaiFeng Dong' \\ HaiTao Xue' \\ JianGang Cheng ${ }^{2}$ \\ Jing Su' \\ Dan $\mathrm{Li}^{1}$ \\ JiHua Zhang' \\ HaoLei Zhang' \\ 'Department of Ear-Nose-Throat, The \\ First Hospital of Hebei Medical \\ University, Shijiazhuang, Hebei 05003I, \\ People's Republic of China; ${ }^{2}$ Department \\ of Ear-Nose-Throat, Shijiazhuang Ping'an \\ Hospital, Shijiazhuang, Hebei 05002I, \\ People's Republic of China
}

Correspondence: HaiTao Xue Department of Ear-Nose-Throat, The First Hospital of Hebei Medical University, Donggang Road No. 89, Yuhua District, Shijiazhuang, Hebei 05003I, People's

Republic of China

Tel +86-3II-859I7072

Email haitxue@I26.com
Introduction: Laryngeal cancer is the most common head and neck cancer worldwide. It is urgent to identify the mechanisms underlying laryngeal cancer pathogenesis. In the present study, we investigated the biological functions of Peripherin 2 (PRPH2) in laryngeal cancer and uncovered the molecular mechanism underlying this disease.

Methods: Laryngeal cancer tissues were used to analyze the expression of PRPH2. In vitro transwell matrigel invasion assay and annexin V anoikis assay in laryngeal cancer cells were conducted to investigate PRPH2 related biological functions. Quantitative real-time PCR and Western blotting were performed to investigate the expression and mechanism of PRPH2 in laryngeal cancer.

Results: We found that the expression of PRPH2 was significantly downregulated in laryngeal cancer tissues. Overexpression of PRPH2 suppressed the invasion and anoikis inhibition of laryngeal cancer cells. Furthermore, PRPH2 overexpression increased the phosphorylation of YAP and LATS1 and decreased the activities of Rho GTPases, while PRPH2 knockdown had opposite effects. Inhibitors of the Hippo pathway abrogated PRPH2 knockdown-induced laryngeal cancer cell invasion and anoikis inhibition.

Discussion: These results suggested that PRPH2 suppresses laryngeal cancer cell invasion and anoikis inhibition by activating Hippo signalling. PRPH2 may serve as a potential therapeutic target for laryngeal cancer in the future.

Keywords: PRPH2, hippo signaling, laryngeal cancer, invasion, anoikis inhibition

\section{Introduction}

Laryngeal cancer is the most common head and neck cancer worldwide. The increased incidence of laryngeal cancer has been reported in recent years. ${ }^{1,2}$ Until recently, conservative surgery and radiotherapy alone or in combination have been advised for the treatment of laryngeal cancer. Thus, there is an urgent need to identify the mechanisms underlying laryngeal cancer pathogenesis. Because invasion and metastasis are the main causes of mortality in patients with solid tumours, these factors have received much attention in recent studies. ${ }^{3-5}$ However, the current knowledge of the molecular mechanisms underlying invasion and metastasis in laryngeal cancer remains scarce. $^{6-8}$

The Hippo signalling pathway plays an important role in regulating the invasion and metastasis of cancer cells. ${ }^{9-11}$ Hippo signalling includes the following kinase cascade. Macrophage Stimulating 1/2 (MST1/2) in coordination with the regulatory protein SAV1 activates Large Tumour Suppressor Kinase 1/2 (LATS1/2), which phosphorylates and inactivates Yes-Associated Protein (YAP)/Tafazzin (TAZ). Then, YAP/TAZ are restrained in the cytoplasm and lose their ability to 
transcriptionally activate related genes. Many biological factors such as contact inhibition, cell polarity/adhesion molecules, and cellular metabolic status can activate Hippo signalling. ${ }^{12,13}$

Peripherin 2 (PRPH2), also known as RDS, was initially identified as a cause of natural retinal degeneration in rats. ${ }^{14}$ Retinal outer segment membrane protein 1 (ROM1) and PRPH2 form complexes through both covalent and noncovalent interactions that are important to the formation and maintenance of photoreceptor outer segments. ${ }^{15-18}$ PRPH2 is a transmembrane glycoprotein that is intrinsic to the curvature formation of each disc and flattened surface morphology. Deficiency of this protein results in cellular disorganization and cellular apoptosis activation via unknown mechanisms. ${ }^{15,19}$ Nevertheless, the link between PRPH2 and Hippo signalling has not been reported.

In the present study, we found that PRPH2 expression was significantly downregulated in laryngeal cancer tissues. The overexpression of PRPH2 could significantly suppress invasion and anoikis inhibition in laryngeal cancer cells. Furthermore, the effects of PRPH2 on the biological behaviours of laryngeal cancer cells were found to be dependent on Hippo signalling activation.

\section{Methods and Materials Cell Culture}

Human laryngeal cancer cell lines, including Hep-2, TU212, TU686, M2e, M4e and AMC-HN-8, were purchased from the Cell Bank of the Chinese Academy of Sciences. Dulbecco's modified Eagle's medium (DMEM) supplemented with $10 \%(\mathrm{v} / \mathrm{v})$ foetal calf serum (FCS) and $1 \%$ antibiotics was used here. The cells were incubated at $37{ }^{\circ} \mathrm{C}$ in a humidified incubator under $5 \% \mathrm{CO}_{2}$ conditions.

\section{Clinical Samples}

Human laryngeal cancer (16 cases) and corresponding normal tissues (12 cases), in which 12 cases were paired, were obtained from the Department of Ear-Nose-Throat, The First Hospital of Hebei Medical University. The human tissue microarray, containing 48 cases of laryngeal cancer samples, was purchased from Alenabio. All the patients were provided with written informed consent before enrollment and in compliance with the Declaration of Helsinki. The study was approved by the by the ethical review committee of the First Hospital of Hebei Medical University (directed by the World Health
Organization Collaborating Centre for Research in Human Production).

\section{Quantitative Real-Time PCR}

Total RNA of cells or tissues was extracted by TRIzol (Takara) and reverse transcribed by the PrimeScript RT-PCR kit (Perfect Real Time). Quantitative real-time PCR analyses were performed with SYBR Premix Ex Taq (Takara) on a 7500 real-time PCR system (Applied Biosystems) at the recommended thermal cycling settings: 1 cycle at $95{ }^{\circ} \mathrm{C}$ for 30 seconds, followed by 40 cycles of 5 seconds at $95^{\circ} \mathrm{C}$ and 31 seconds at $60{ }^{\circ} \mathrm{C}$. The primer sequences used were: PRPH2, forward 5'-CAGAAGAAGCGGGTCAAGTTG-3' and reverse 5'-GCTCCTCTTTCGGAGTTCAATC-3'; CTG F, forward 5'-TGGAGATTTTGGGAGTACGG-3' and reverse 5'-CAGGCTAGAGAAGCAGAGCC-3'; ANKRD1, forward 5'-GTGTAGCACCAGATCCATCG-3' and reverse 5'- CGGTGAGACTGAACCGCTAT-3'; CYR61, forward 5'CCCGTTTTGGTAGATTCTGG-3' and reverse 5'-GCTGGA ATGCAACTTCGG-3'; and $\beta$-actin, forward 5'-CTCCATC CTGGCCTCGCTGT-3' and reverse 5'-GCTGTCACCTTC ACCGTTCC-3'.

\section{Western Blotting and GTPase Pull-Down Assays}

The cells were lysed in lysis buffer, and the proteins were separated by SDS-PAGE under reducing conditions. The membrane was blocked in phosphate-buffered saline/Tween20 containing 5\% BSA. Then, antibodies against PRPH2 (Abcam), phospho-YAP (Cell Signalling), YAP (Cell Signalling), phospho-LATS1 (Cell Signalling), LATS1/2 (Cell Signalling), GAPDH (Sigma) and species-specific secondary antibodies were used to incubate the membrane. The secondary antibodies were detected by the Odyssey imaging system (LI-COR). GTPase pull-down assays were performed according to standard procedures as previously described. ${ }^{20}$

\section{Lentivirus Production and Cell Transduction}

The 293 T cells were cotransfected with the $\mathrm{pEZ-lv105}$ vector (GeneCopoeia) by using Lipofectamine 2000 (Invitrogen) for virus packaging. The viruses were harvested at 24, 48 and $72 \mathrm{hrs}$ after transfection. After detecting the virus titres, $1 \times 10^{5}$ cells were infected with $1 \times 10^{6}$ recombinant lentivirustransducing units by using $5 \mu \mathrm{g} / \mathrm{mL}$ of polybrene (Sigma). 


\section{siRNA Transfection}

Small interfering RNAs duplexes targeting PRPH2 were produced by Genepharma. The transfection steps were performed according to the manufacturer's protocols.

\section{Hippo Inhibitors}

Inhibitors of Hippo (Verteporfin and Peptide 17) were purchased from Selleck. Verteporfin (chemical formula: C41H42N4O8) and Peptide17 (XMU-MP-1, chemical formula: $\mathrm{C} 93 \mathrm{H} 144 \mathrm{ClN} 23 \mathrm{O} 21 \mathrm{~S} 2$ ) are small molecular compounds that can inhibit the interaction of YAP-TEAD. ${ }^{21,22}$ To inhibit the interaction of YAP-TEAD, Verteporfin and Peptide 17 were added into M2e or AMC-HN-8 cells infected with siRNA of PRPH2 or NC. All cells were incubated at $37^{\circ} \mathrm{C}$.

\section{Invasion Assay}

Laryngeal cancer cells were detached and resuspended in serum-free DMEM medium. The cells were plated at $2 \times 10^{4}$ cells in $0.1 \mathrm{~mL}$ onto Matrigel (BD)-coated inserts (Millipore) seated on a 24-well plate. Then, 5\% FBS DMEM medium was added to the bottom chamber. The cells were incubated at $37^{\circ} \mathrm{C}$. After $48 \mathrm{hrs}$, the filters were fixed and stained with $0.1 \%(\mathrm{w} / \mathrm{v})$ Crystal Violet. Noninvading cells were removed, and invading cells were counted under a microscope at 400× magnification. Three grids per field were counted, and the experiments were repeated twice.

\section{Anoikis Assays}

For these assays, $5 \times 10^{5}$ Laryngeal cancer cells were cultured on poly-HEMA-treated 12-well plates for $48 \mathrm{hrs}$ at $37{ }^{\circ} \mathrm{C}$ in a $5 \% \mathrm{CO}_{2}$ atmosphere. After incubation, adherent cells were detached with $0.25 \%$ trypsin $/ 0.01 \%$ EDTA in $1 \times$ PBS. Detached and suspended cells were harvested in complete DMEM medium and centrifuged at $1000 \mathrm{rpm} /$ 5 mins. The cells were washed with $1 \times$ PBS and stained with $100 \mu \mathrm{l}$ binding buffer containing $1.75 \mu \mathrm{g} / \mathrm{mL}$ Annexin $\mathrm{V}$ and $1.75 \mu \mathrm{g} / \mathrm{mL}$ propidium iodide (PI). The cells were incubated at room temperature for $15 \mathrm{mins}$ and analysed by flow cytometry (BD).

\section{Statistical Analysis}

Data were presented as the means \pm standard error of the mean (SEM). Student's $t$-test and one-way ANOVA were used for comparisons between groups. Values of $\mathrm{P}<0.05$ were considered statistically significant.

\section{Results}

PRPH2 Is Significantly Downregulated in Laryngeal Cancer Tissues, and PRPH2 Expression Is Suppressed by Epigenetic Methylation

To investigate the expression level of PRPH2 in laryngeal cancer tissues, we collected tissues from 16 laryngeal cancer cases and 12 normal cases. Quantitative real-time PCR revealed that the expression of PRPH2 was significantly downregulated in laryngeal cancer tissues (Figure 1A). Among 12 paired laryngeal cancer and normal tissues, PRPH2 expression was also significantly downregulated in laryngeal cancer tissues (Figure 1B). Human laryngeal cancer tissue microarray $(\mathrm{n}=48)$ showed that the expression of PRPH2 was downregulated in $67.80 \%$ of laryngeal cancer tissues compared to normal tissues (Figure 1C).

Furthermore, we investigated the reason for PRPH2 downregulation in laryngeal cancer. Notably, after treatment with 5-aza-20-deoxycytidine (DAC), a specific methyltransferase inhibitor, and trichostatin A (TSA), a histone deacetylase inhibitor, methylation was observed in all 3 tested laryngeal cancer cell lines (TU686, M4E and TU686 cells), and histone acetylation was observed in 1 cell line. These results suggested that epigenetic methylation is the main cause of the suppression of PRPH2 expression in laryngeal cancer (Figure 1D-F).

\section{Overexpression of PRPH2 Suppresses Invasion and Anoikis Inhibition in Laryngeal Cancer Cells}

To further investigate the biological functions of PRPH2 in laryngeal cancer, we detected the expression level of PRPH2 in 6 laryngeal cancer cell lines. As shown in Figure 2A, the expression of PRPH2 in TU686 and M4e cells was obviously lower than that in other laryngeal cancer cell lines. We further established stable PRPH2-overexpressing TU686 and M4e cell lines by the transduction of lentivirus carrying the PRPH2 gene named Lenti-PRPH2. Western blot analyses revealed that PRPH2 was successfully overexpressed in both TU686 (Figure 2B) and M4e (Figure 2C) cells.

We first investigated the role of PRPH2 in the invasion of laryngeal cancer cells. By Transwell Matrigel invasion assay, we found that the overexpression of PRPH2 suppressed the invasion of TU686 and M4e cells (Figure 2D and F) after $48 \mathrm{hrs}$. Furthermore, as shown by annexin V anoikis assay, 
A

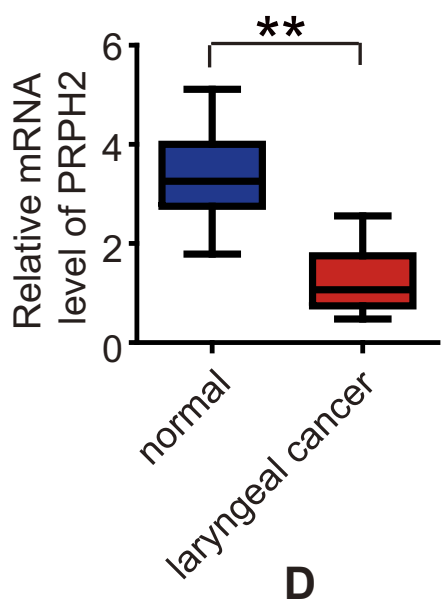

B

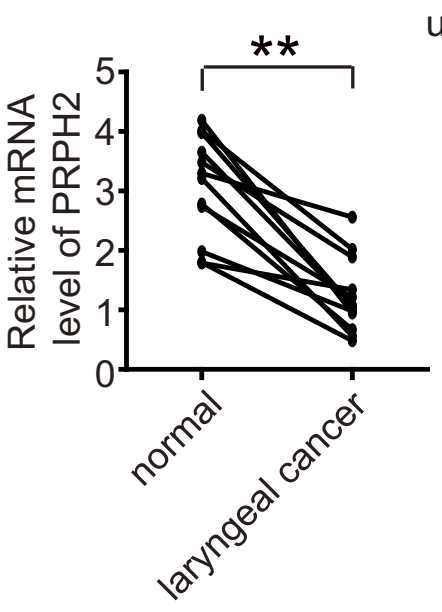

C

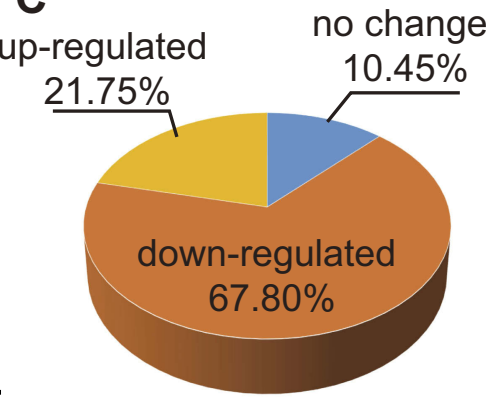

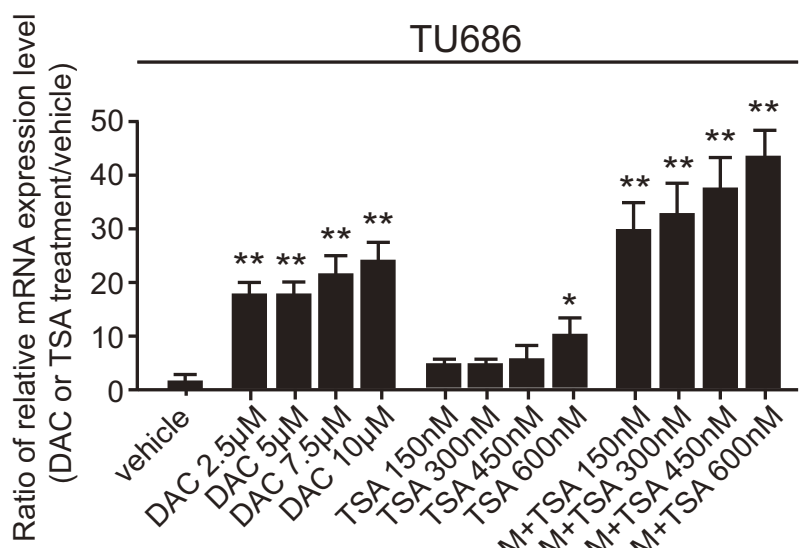

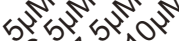

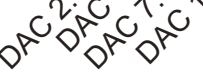

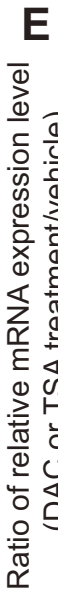

E

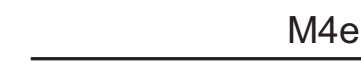

\section{M4e}
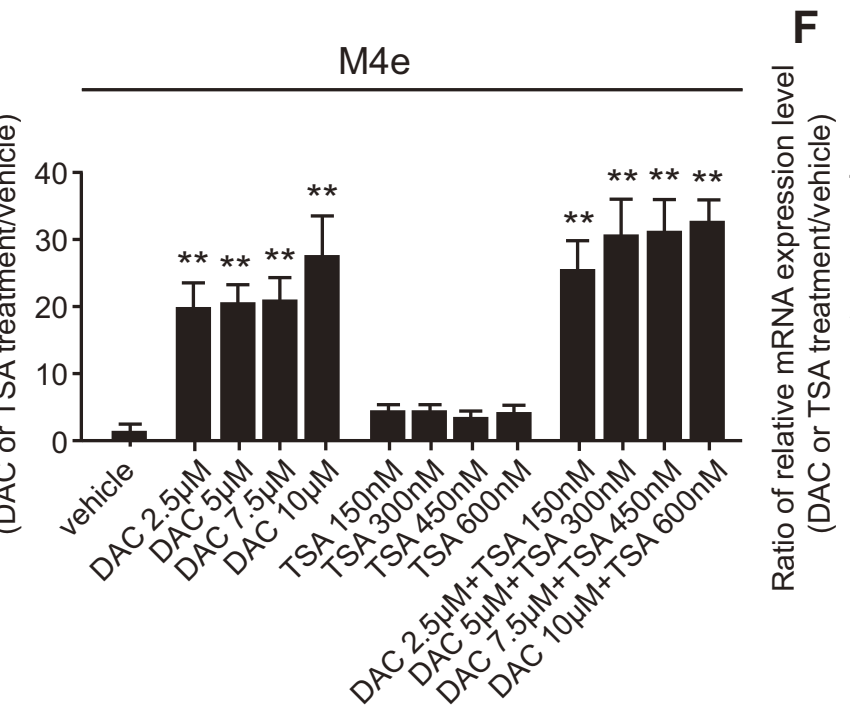

Hep-2

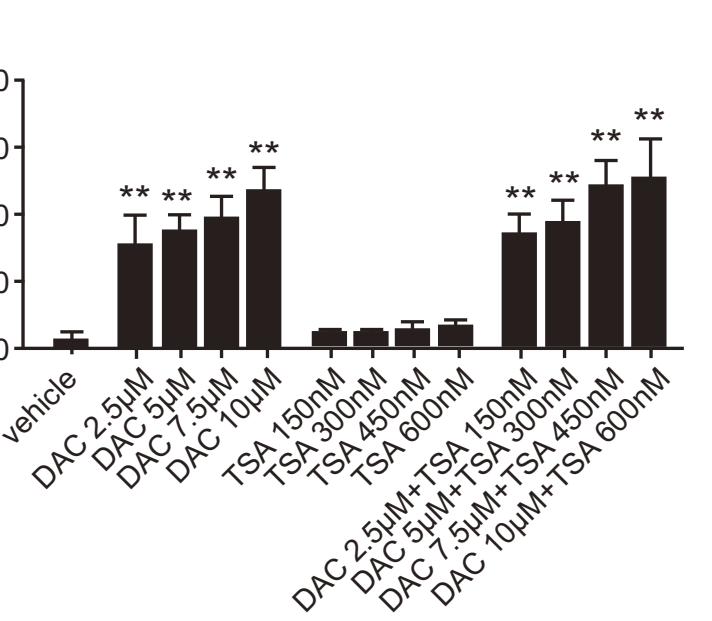

Figure I The expression of PRPH2 is downregulated in laryngeal cancer tissues, and DNA methylation suppresses PRPH2 expression. (A) The mRNA expression of PRPH2 in 16 cases of laryngeal cancer $(n=16)$ and 12 cases of normal tissues $(n=12)$. $* * P<0.01$, Laryngeal cancer vs. normal tissues. (B) The mRNA expression of PRPH2 in I2 paired laryngeal cancer and normal tissues. ${ }^{* * P}<0.0 \mathrm{I}$, Laryngeal cancer vs. normal tissues $(\mathrm{n}=12$ in each group). (C) The expression of PRPH2 was downregulated in $67.80 \%$ laryngeal cancer tissues $(n=48)$. (D-F) Relative mRNA expression of PRPH2 in TU686 (D), M4e (E) and Hep-2 (F) cells after treatment with DAC and TSA (repeat 3 times for each group). $* P<0.05$, TSA $600 \mathrm{nM}$ vs. vehicle in TU686 cells; $* * P<0.01$, DAC vs. vehicle, DAC+TSA vs. vehicle in TU686, M4E and TU686 cells. 


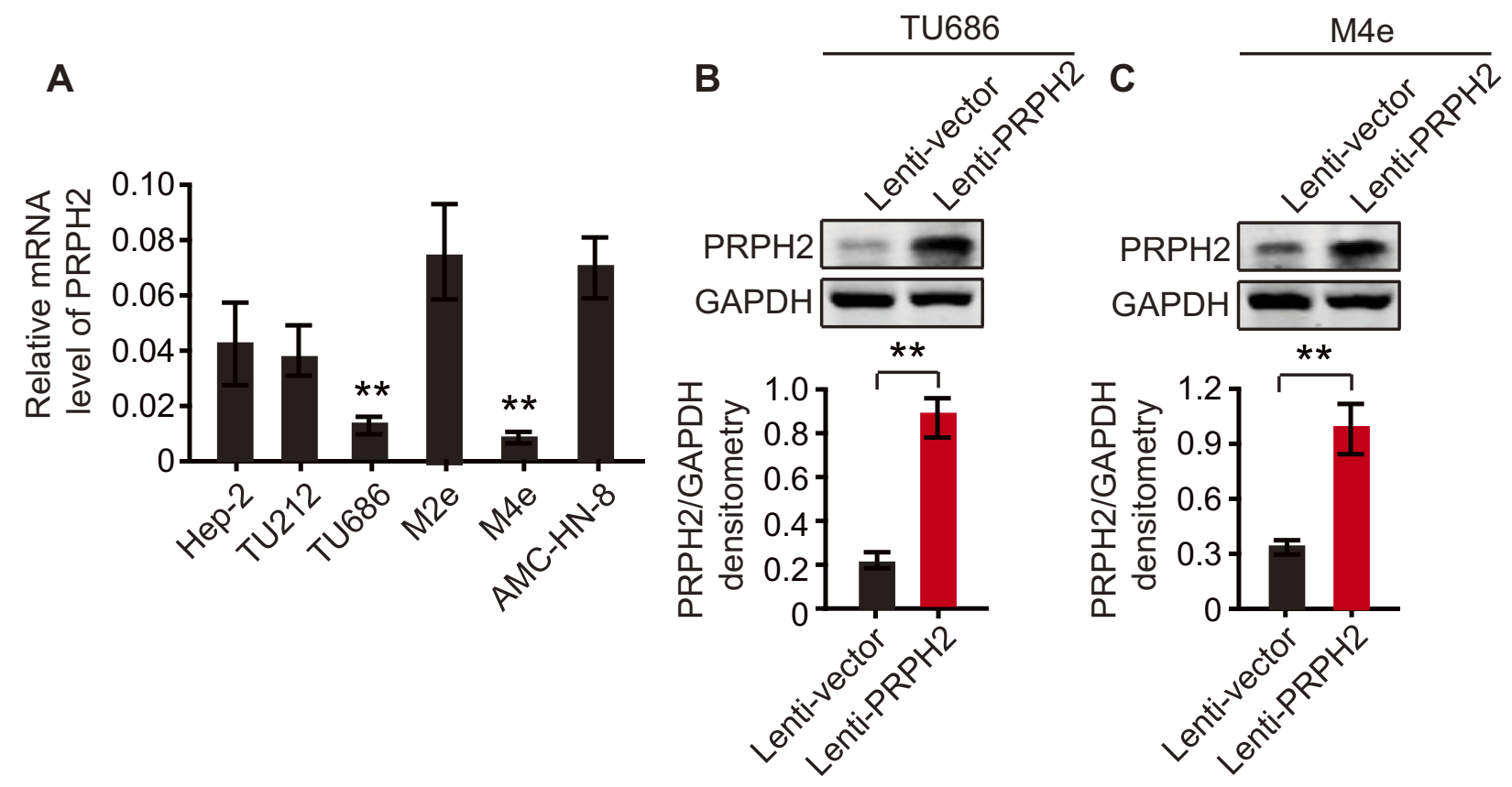

D

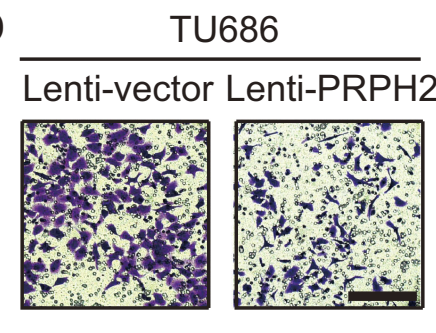

$\mathbf{F}$

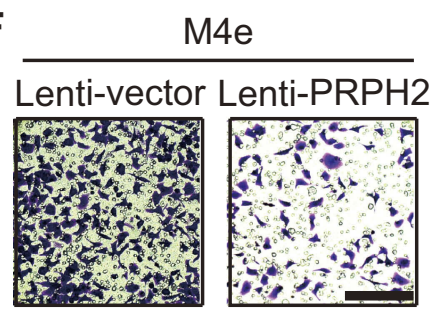

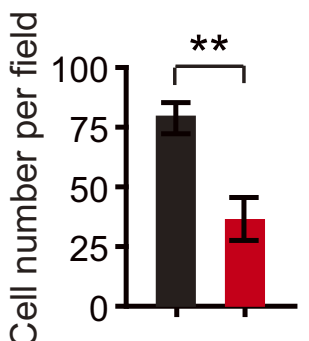
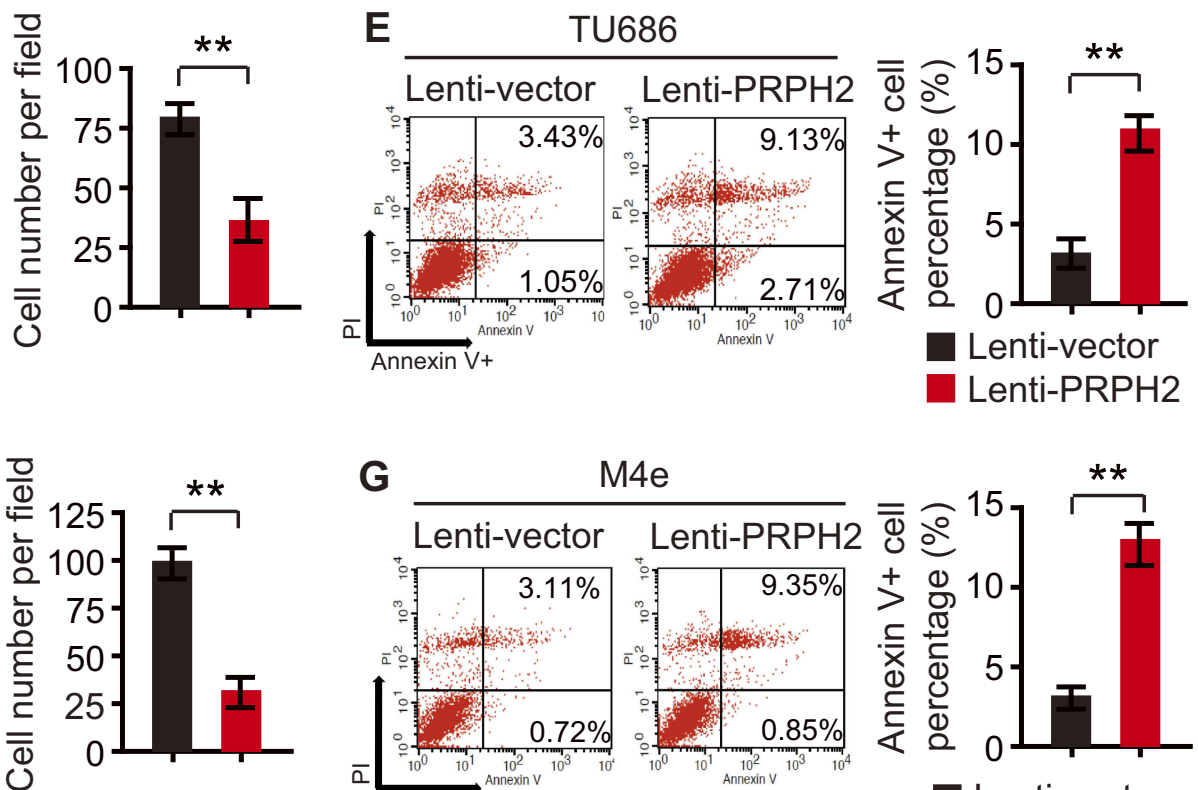
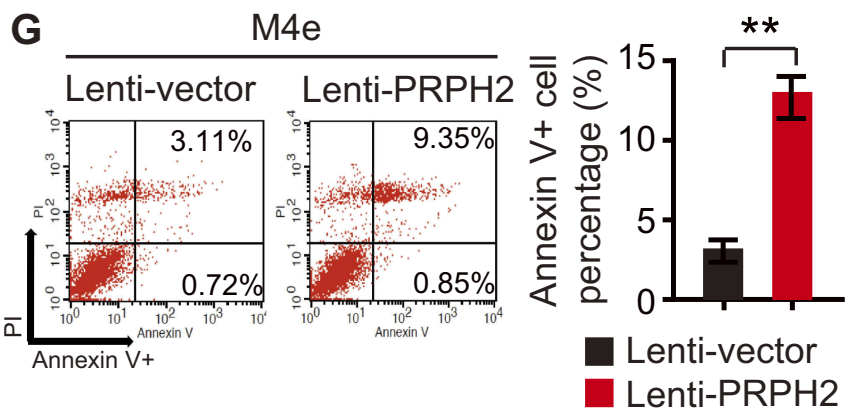

Figure 2 Overexpression of PRPH2 suppresses the invasion and anoikis inhibition of laryngeal cancer cells. (A) Expression of PRPH2 in laryngeal cancer cell lines, including Hep-2, TU2 2, TU686, M2e, M4e and AMC-HN-8 cells (repeat 3 times for each group). ${ }^{* * P<0.01, ~ T U 686 ~ o r ~ M 4 E ~ v s . ~ M 2 e ~ o r ~ A M C-H N-8 . ~(B ~ a n d ~ C) ~ T h e ~ p r o t e i n ~ e x p r e s s i o n ~}$ levels of PRPH2 in TU686 (B) and M4E (C) cells, infected with Lenti-PRPH2 or Lenti-vector. Statistical analyses of PRPH2 expression in the two groups are shown below (repeat 3 times for each group). ${ }^{* * P}<0.01$, Lenti-vector vs. Lenti-PRPH2. (D) Representative photos of invaded TU686 cells infected with Lenti-PRPH2 or Lenti-vector. Statistical analysis of invaded TU686 cells in the two groups is shown right (repeat 3 times for each group). $* * P<0.01$, Lenti-vector vs. Lenti-PRPH2. (E) Flow cytometry analysis of anoikis of TU686 cells infected with Lenti-PRPH2 or Lenti-vector. Flow cytometry statistical analysis of apoptotic TU686 cells is shown right (repeat 3 times for each group). ${ }^{*} * \mathrm{P}<0.01$, Lenti-vector vs. Lenti-PRPH2. (F) Representative photos of invaded M4e cells infected with Lenti-PRPH2 or Lenti-vector. Statistical analysis of invaded M4e cells in the two groups is shown right (repeat 3 times for each group). ${ }^{* * P}<0.01$, Lenti-vector vs. Lenti-PRPH2. (G) Flow cytometry analysis of anoikis of M4e cells infected with Lenti-PRPH2 or Lenti-vector. Flow cytometry statistical analysis of apoptotic M4e cells is shown right (repeat 3 times for each group). $* * P<0.01$, Lenti-vector vs. Lenti-PRPH2. 
we found that the overexpression of PRPH2 promoted anoikis in TU686 and M4e cells (Figure 2E and G) after 48 hrs.

\section{PRPH2 Overexpression Increases the Phosphorylation of YAP/LATSI Signalling and Decreases Rho GTPase Activities}

To investigate the underlying mechanism of the association of PRPH2 with laryngeal cancer, we performed GESA analysis and found that PRPH2 was closely related to the Hippo signalling pathway (Figure 3A). Western blot analyses revealed that the Hippo signalling pathway plays important roles in laryngeal cancer invasion and metastasis. Interestingly, the overexpression of PRPH2 in TU686 cells significantly increased the phosphorylation of YAP and LATS1 (Figure 3B). Then, PRPH2-overexpressing and control TU686 cells were serum starved for $24 \mathrm{~h}$. As shown by pull-down assays, the activities of RhoA and $\mathrm{Cdc} 42$ were found to be significantly suppressed by PRPH2 overexpression (Figure 3C).

We also detected the mRNA levels of the following canonical target genes of YAP: Connective Tissue Growth Factor (CTGF), Ankyrin Repeat Domain 1 (ANKRD1), and Cysteine Rich Angiogenic Inducer 61 (CYR61). Additionally, CTGF, ANKRD1 and CYR61 mRNA levels were significantly suppressed in PRPH2-overexpressing TU686 cells (Figure 3D). These results demonstrated that the Hippo pathway was activated in PRPH2-overexpressing laryngeal cancer cells, which suppressed laryngeal cancer cell invasion and anoikis inhibition.

\section{Knockdown of PRPH2 Promotes Invasion} and Anoikis Inhibition in Laryngeal Cancer Cells, and These Effects Can Be Abrogated by Hippo Pathway Inhibitors

Furthermore, we knocked down PRPH2 in M2e and AMC-HN-8 cells, which express high levels of PRPH2, by using siRNAs (called si-PRPH2-1 and si-PRPH2-2). Western blot analyses revealed that PRPH2 was silenced in M2e (Figure 4A) or AMC-HN-8 (Figure 4B) cells. Transwell Matrigel invasion and annexin $\mathrm{V}$ anoikis assays showed that the knockdown of PRPH2 could promote invasion and anoikis inhibition in laryngeal cancer cells (Figure 4C-F).

By the administration of verteporfin (an inhibitor of YAP-TEAD) and peptide 17 (YAP-TEAD inhibitor I, an inhibitor of YAP-TEAD), we further investigated the correlation between PRPH2 and Hippo signalling. Verteporfin and peptide 17 were added to PRPH2-silenced and control M2e and AMC-HN-8 cells. Verteporfin and peptide 17 abrogated PRPH2 knockdown-induced M2e and AMC-HN-8 cell invasion (Figure 4C and E). Moreover, anoikis inhibition in M2e and AMC-HN-8 cells induced by PRPH2 knockdown was also abrogated by these inhibitors (Figure 4D and F).

These results indicated that PRPH2 suppresses laryngeal cancer cell invasion and anoikis inhibition by activating Hippo signalling (Figure 4G).

\section{Discussion}

Few studies on PRPH2 in cancers, particularly those on the detailed biological functions and related mechanism of PRPH2 in laryngeal cancer, have been reported in recent years. In the present study, the precise roles of PRPH2 in laryngeal cancer were investigated for the first time. The downregulated expression of PRPH2 may lead to the development and progression of laryngeal cancer. The biological function experiments revealed that PRPH2 overexpression could suppress the invasion and anoikis inhibition of laryngeal cancer cells, which indicated that PRPH2 is involved in the invasion and anoikis inhibition of laryngeal cancer.

Invasion and metastasis are major concerns during the prognosis and progression of cancer. Hippo signalling is very important in the invasion and metastasis of cancer cells. $^{9-11}$ YAP contributes to metastasis via multiple mechanisms. YAP interacts with TEAD and FOS in the nucleus to reprogram gene expression to induce epithelialmesenchymal transition (EMT). YAP also antagonizes E-cadherin junction assembly by regulating actin cytoskeleton organization and contributes to EMT. Furthermore, YAP activation promotes stiffening of the extracellular matrix of cancer-associated fibroblasts (CAFs) to enhance YAP nuclear localization in cancer cells. Such interplay between cancer cells and CAFs might amplify the effects of YAP during tumorigenesis. ${ }^{23-26}$

In the present study, the overexpression of PRPH2 increased the phosphorylation of YAP and LATS1, restraining these proteins in the cytoplasm and inhibiting their transcriptional activation. Furthermore, these results were confirmed by the detection of Rho GTPase activities and canonical YAP target gene expression. Meanwhile, knockdown of PRPH2 lead to an opposite effect. By using inhibitors of the Hippo pathway, it was found that PRPH2 knockdown induced laryngeal cancer cell invasion, and 

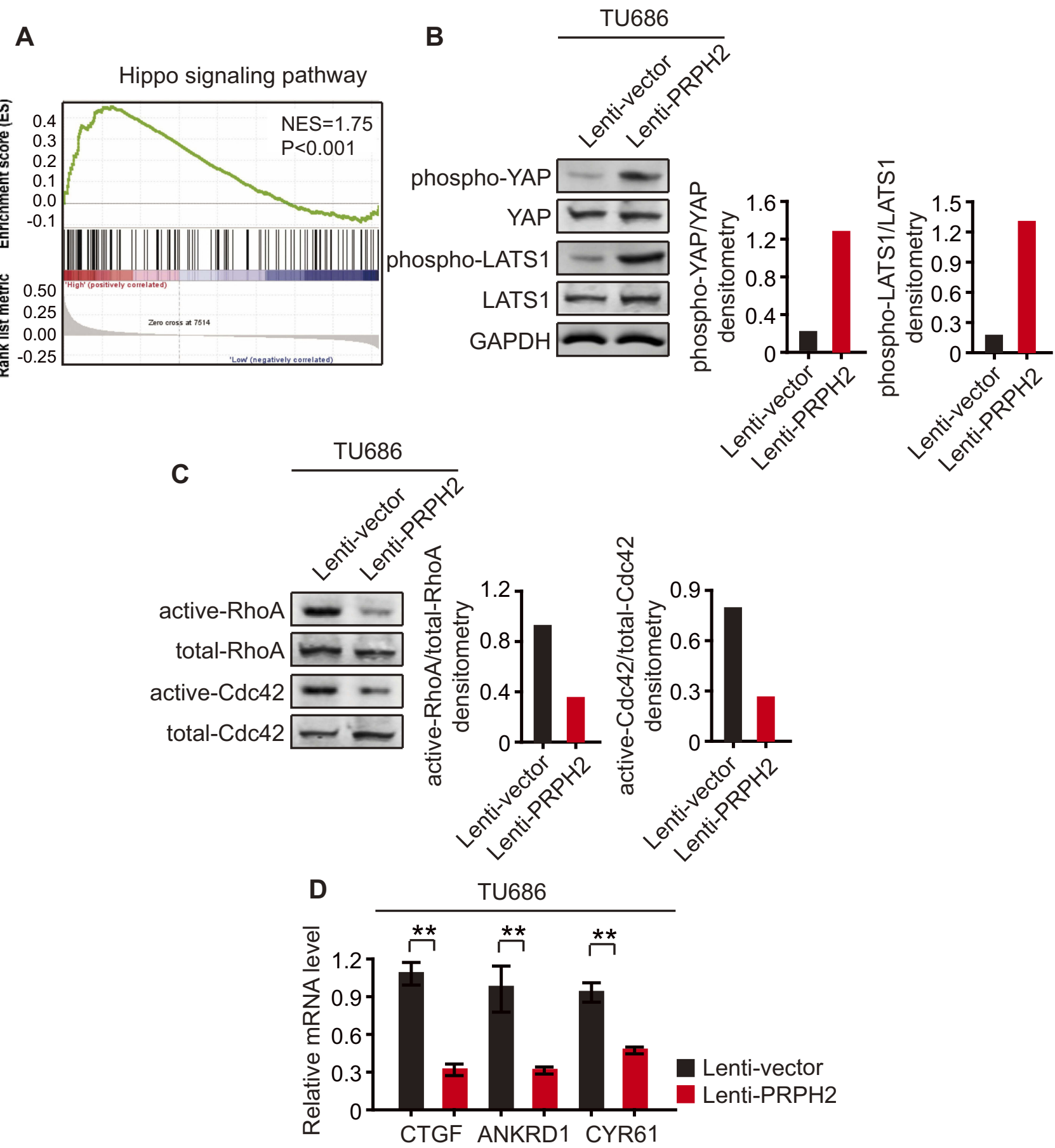

Figure 3 PRPH2 overexpression increases the phosphorylation of YAP/LATSI and decreases Rho GTPases activities. (A) GESA analysis showed that PRPH2 was closely related with Hippo signalling pathway. (B) Western blot analysis of phospho-YAP, YAP, phospho-LATSI and LATSI in PRPH2 overexpressed and control TU686 cells (repeat 3 times for each group). Phospho-YAP/YAP and phospho-LATSI/LATSI densitometry are shown on the right. (C) TU686 cells were serum starved for $24 \mathrm{~h}$ and the RhoA and Cdc42 activities were measured by pull-down assays in PRPH2 overexpressed and control cells (repeat 3 times for each group). Active-RhoA/total-RhoA and activeCdc42/total-Cdc42 densitometry are shown on the right. (D) The mRNA levels of CTGF, ANKRDI and CYR6I in PRPH2 overexpressed and control TU686 cells (repeat 3 times for each group). $* * P<0.01$, Lenti-vector vs. Lenti-PRPH2.

anoikis inhibition could be abrogated by these inhibitors, indicating that PRPH2 suppressed laryngeal cancer cell invasion and anoikis inhibition by activating Hippo signalling pathway.
In conclusion, the results of the present study showed that PRPH2 plays an important role in laryngeal cancer cell invasion and anoikis inhibition. The overexpression of PRPH2 in laryngeal cancer cells suppresses Rho GTPase 


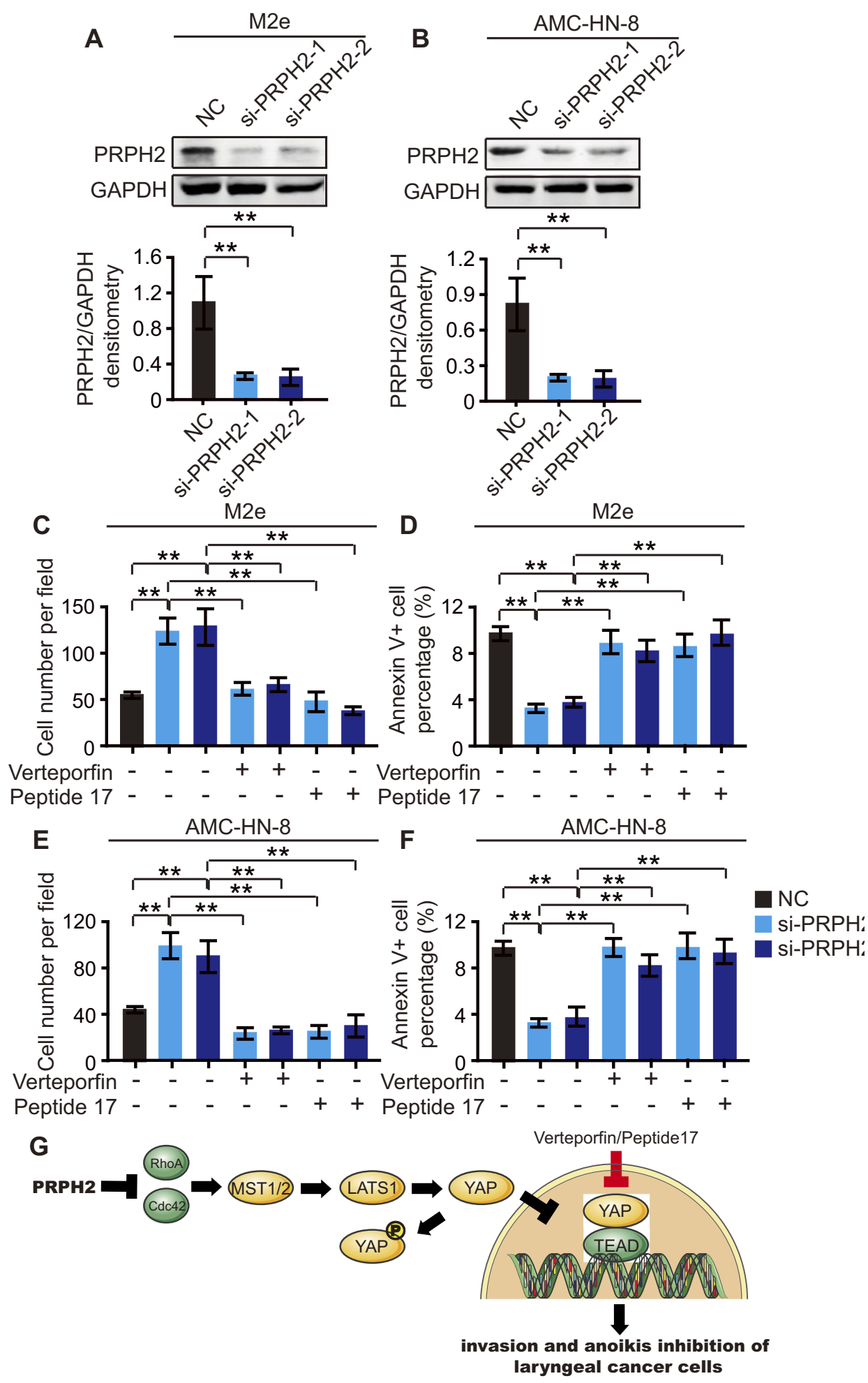

Figure 4 PRPH2 knockdown promotes the invasion and anoikis inhibition of laryngeal cancer cells, which can be abrogated by Hippo pathway inhibitors. (A and B) The protein expression level of PRPH2 in M2e (A) and AMC-HN-8 (B) cells, infected with siRNA of PRPH2 or negative control (NC). Statistical analyses of PRPH2 expression are shown below (repeat 3 times for each group). ${ }^{* * P}<0.01, \mathrm{NC}$ vs. si-PRPH2-I or si-PRPH2-2. (C and E) M2e and AMC-HN-8 cells were infected with siRNA of PRPH2 or NC, then treated with $50 \mathrm{nM}$ Verteporfin (YAP-TEAD inhibitor) or $50 \mathrm{nM}$ Peptide 17 (YAP-TEAD inhibitor), respectively. The invaded M2e (C) and AMC-HN-8 (E) cells were analysed after 48 hrs (repeat 3 times for each group). $* * P<0.01$, NC vs. si-PRPH2-I or si-PRPH2-2, si-PRPH2-I vs. si-PRPH2-I+Veterporfin or si-PRPH2-I+Peptide I7, si-PRPH2-2 vs. si-PRPH2-2+Veterporfin or si-PRPH2 -2+Peptide 17. (D and F) M2e and AMC-HN-8 cells were infected with siRNA of PRPH2 or NC, then treated with $50 \mathrm{nM}$ Verteporfin or $50 \mathrm{nM}$ Peptide 17, respectively. The apoptotic M2e (D) and AMC-HN-8 (F) cells were analysed after 48 hrs (repeat 3 times for each group). $* * P<0.01$, NC vs. si-PRPH2-I or si-PRPH2-2, si-PRPH2-I vs. si-PRPH2-I+Veterporfin or si-PRPH2-I+Peptide 17, si-PRPH2-2 vs. si-PRPH2-2+Veterporfin or si-PRPH2-2+Peptide 17. (G) PRPH2 decreases Rho GTPases activities, and subsequently increases the phosphorylation of YAP/LATSI and activates Hippo signalling, thereby suppressing laryngeal cancer cell invasion and anoikis inhibition. 
activities, activates Hippo signalling, and suppresses invasion and anoikis inhibition in laryngeal cancer cells. The use of PRPH2 may be a future therapeutic strategy for laryngeal cancer.

\section{Acknowledgements}

This study was funded by Hebei Province Natural Science Foundation.

\section{Disclosure}

The authors report no conflicts of interest in this work.

\section{References}

1. Chu EA, Kim YJ. Laryngeal cancer: diagnosis and preoperative work-up. Otolaryngol Clin North Am. 2008;41:673-695. doi:10.1016/j4tc.2008.01.016

2. Fu Q, Liu P, Sun X, et al. Ribonucleic acid interference knockdown of IL-6 enhances the efficacy of cisplatin in laryngeal cancer stem cells by down-regulating the IL-6/STAT3/HIF1 pathway. Cancer Cell Int. 2017;17:79. doi:10.1186/s12935-017-0448-0

3. Gupta GP, Massagué J. Cancer metastasis: building a framework. Cell. 2006;127:679-695. doi:10.1016/j.cell.2006.11.001

4. Fidler IJ. The pathogenesis of cancer metastasis: the 'seed and soil' hypothesis revisited. Nat Rev Cancer. 2003;3:453-458. doi:10.1038/ nrc1098

5. Wang J, Ge W, Wang Y, et al. MiR-613 suppressed the laryngeal squamous cell carcinoma progression through regulating PDK1. J Cell Biochem. 2017. doi:10.1002/jcb.26468

6. Rothman KJ, Cann CI, Flanders D, et al. Epidemiology of laryngeal cancer. Epidemiol Rev. 1980;2:195-209. doi:10.1093/oxfordjournals. epirev.a036223

7. Siegel R, Naishadham D, Jemal A. Cancer statistics, 2013. $C A$ Cancer J Clin. 2013;63:11-30. doi:10.3322/caac.v63.1

8. Tamaki A, Miles BA, Lango M, et al. AHNS series: do you know your guidelines? Review of current knowledge on laryngeal cancer Head Neck. 2017. doi:10.1002/hed.24862

9. Han Q, Lin X, Zhang X, et al. WWC3 regulates the Wnt and hippo pathways via dishevelled proteins and large tumour suppressor 1 , to suppress lung cancer invasion and metastasis. $J$ Pathol. 2017;242:435-447. doi:10.1002/path.2017.242.issue-4

10. Tong R, Yang B, Xiao H, et al. KCTD11 inhibits growth and metastasis of hepatocellular carcinoma through activating hippo signaling. Oncotarget. 2017;8:37717-37729. doi:10.18632/oncotarget.v8i23

11. Varzavand A, Hacker W, Ma D, et al. $\alpha 3 \beta 1$ integrin suppresses prostate cancer metastasis via regulation of the hippo pathway. Cancer Res. 2016;76:6577-6587. doi:10.1158/0008-5472.CAN-161483
12. van Rensburg HJJ, Yang X. The roles of the hippo pathway in cancer metastasis. Cell Signal. 2016;28:1761-1772. doi:10.1016/j. cellsig.2016.08.004

13. Juan WC, Hong W. Targeting the hippo signaling pathway for tissue regeneration and cancer therapy. Genes. 2016;7:55. doi:10.3390/ genes 7090055

14. Démant $P$, Iványi $D$, van Nie $R$. The map position of the rds gene on the 17th chromosome of the mouse. Tissue Antigens. 1979;13:53-55. doi:10.1111/j.1399-0039.1979.tb01136.x

15. Stuck MW, Conley SM, Naash MI. Retinal degeneration slow (RDS) glycosylation plays a role in cone function and in the regulation of RDS.ROM-1 protein complex formation. $J$ Biol Chem. 2015;290:27901-27913. doi:10.1074/jbc.M115.683698

16. Zulliger R, Conley SM, Mwoyosvi ML, et al. Oligomerization of Prph2 and Rom1 is essential for photoreceptor outer segment formation. Hum Mol Genet. 2018;27:3507-3518. doi:10.1093/hmg/ ddy 240

17. Conley SM, Stuck MW, Watson JN, et al. Prph2 initiates outer segment morphogenesis but maturation requires $\operatorname{Prph} 2 / \mathrm{Rom} 1$ oligomerization. Hum Mol Genet. 2019;28:459-475. doi:10.1093/ $\mathrm{hmg} / \mathrm{ddy} 359$

18. Stuck MW, Conley SM, Naash MI. PRPH2/RDS and ROM-1: historical context, current views and future considerations. Prog Retin Eye Res. 2016;52:47-63. doi:10.1016/j.preteyeres.2015.12.002

19. Chakraborty D, Conley SM, Al-Ubaidi MR, et al. Initiation of rod outer segment disc formation requires RDS. PLoS One. 2014;9: e98939. doi:10.1371/journal.pone.0098939

20. Zhang Z, Chometon G, Wen T, et al. Migration of epithelial cells on laminins: RhoA antagonizes directionally persistent migration. Eur J Cell Biol. 2011;90:1-12. doi:10.1016/j.ejcb.2010.09.005

21. Schmidt-Erfurth U, Hasan T. Mechanisms of action of photodynamic therapy with verteporfin for the treatment of age-related macular degeneration. Surv Ophthalmol. 2000;45:195-214. doi:10.1016/ S0039-6257(00)00158-2

22. Zhang Z, Lin Z, Zhou Z, et al. Structure-based design and synthesis of potent cyclic peptides inhibiting the YAP-TEAD protein-protein interaction. ACS Med Chem Lett. 2014;5:993-998. doi:10.1021/ $\mathrm{m} 1500160 \mathrm{~m}$

23. Salcedo Allende MT, Zeron-Medina J, Hernandez J, et al. Overexpression of yes associated protein 1, an independent prognostic marker in patients with pancreatic ductal adenocarcinoma, correlated with liver metastasis and poor prognosis. Pancreas. 2017;46:913-920. doi:10.1097/MPA.0000000000000867

24. Qiao Y, Chen J, Lim YB, et al. YAP regulates actin dynamics through ARHGAP29 and promotes metastasis. Cell Rep. 2017;19:1495-1502. doi:10.1016/j.celrep.2017.04.075

25. Li C, Wang S, Xing Z, et al. A ROR1-HER3-lncRNA signalling axis modulates the hippo-YAP pathway to regulate bone metastasis. Nat Cell Biol. 2017;19:106-119. doi:10.1038/ncb3464

26. Nokin MJ, Durieux F, Peixoto P, et al. Methylglyoxal, a glycolysis side-product, induces Hsp90 glycation and YAP-mediated tumor growth and metastasis. Elife. 2016;5:e19375. doi:10.7554/eLife. 19375
Cancer Management and Research is an international, peer-reviewed open access journal focusing on cancer research and the optimal use of preventative and integrated treatment interventions to achieve improved outcomes, enhanced survival and quality of life for the cancer patient.
The manuscript management system is completely online and includes a very quick and fair peer-review system, which is all easy to use. Visit http://www.dovepress.com/testimonials.php to read real quotes from published authors. 